University of Wollongong

Research Online

Faculty of Engineering and Information

Faculty of Engineering and Information

Sciences - Papers: Part A

Sciences

$1-1-2013$

\title{
A simple adaptive STFC MB-OFDM UWB system for WBAN applications
}

Miftadi Sudjai

University of Wollongong, ms917@uowmail.edu.au

Le Chung Tran

University of Wollongong, Ictran@uow.edu.au

Farzad Safaei

University of Wollongong, farzad@uow.edu.au

Follow this and additional works at: https://ro.uow.edu.au/eispapers

Part of the Engineering Commons, and the Science and Technology Studies Commons

Research Online is the open access institutional repository for the University of Wollongong. For further information contact the UOW Library: research-pubs@uow.edu.au 


\title{
A simple adaptive STFC MB-OFDM UWB system for WBAN applications
}

\begin{abstract}
This paper proposes a simple, but efficient, adaptive space-time-frequency coded (STFC) multiband orthogonal frequency division multiplexing ultra-wideband (MB-OFDM UWB) system to improve the average BER performance for body-to-external link wireless body area network (WBAN) applications. The novelty of this paper is the proposed body direction based adaptive algorithm that controls the set of modulation scheme, space-time-frequency code (STFC) coding rate, and power of signal constellations implemented in STFC MB-OFDM UWB WBAN. Simulation results show that the proposed system can provide a consistent 1-2 dB improvement in the case of 2/10 configuration, and 1-3 dB improvement in a medium-to-high SNR region in the case of 2120 configuration, compared to the non-adaptive system. The improvement practically means a possible reduction of $12.5 \%-50 \%$ of the total transmitted power to achieve the similar performance as the non-adaptive system. In other words, it can save significantly the power consumption and prolong the battery life of WBAN devices.
\end{abstract}

\section{Keywords}

applications, wban, system, stfc, uwb, mb, ofdm, simple, adaptive

Disciplines

Engineering | Science and Technology Studies

\section{Publication Details}

M. Sudjai, L. Chung. Tran \& F. Safaei, "A Simple Adaptive STFC MB-OFDM UWB System for WBAN Applications," in IEEE International Symposium on Communications and Information Technologies (ISCIT 2013), 2013, pp. 60-65. 


\title{
A Simple Adaptive STFC MB-OFDM UWB System for WBAN Applications
}

\author{
Miftadi Sudjai", Le Chung Tran, and Farzad Safaei \\ School of Electrical, Computer, and Telecommunication Engineering \\ University of Wollongong, NSW 2522 Australia \\ *Email: ms917@,uow.edu.au
}

\begin{abstract}
This paper proposes a simple, but efficient, adaptive space-time-frequency coded (STFC) multiband orthogonal frequency division multiplexing ultra-wideband (MB-OFDM UWB) system to improve the average BER performance for body-to-external link wireless body area network (WBAN) applications. The novelty of this paper is the proposed body direction based adaptive algorithm that controls the set of modulation scheme, space-time-frequency code (STFC) coding rate, and power of signal constellations implemented in STFC MB-OFDM UWB WBAN. Simulation results show that the proposed system can provide a consistent 1-2 dB improvement in the case of $2 \mathrm{I} 10$ configuration, and $1-3 \mathrm{~dB}$ improvement in a medium-to-high SNR region in the case of $2 I 2 O$ configuration, compared to the non-adaptive system. The improvement practically means a possible reduction of $12.5 \%-50 \%$ of the total transmitted power to achieve the similar performance as the non-adaptive system. In other words, it can save significantly the power consumption and prolong the battery life of WBAN devices.
\end{abstract}

Keywords-Adaptive system, MIMO, UWB, WBAN

\section{INTRODUCTION}

Wireless body area network (WBAN) has become the focal point of research during the last years. By utilizing tinylow power and high data rate devices, it promises a wide range of applications, such as entertainment, sports and training, military, and health care [7]. In particular, WBAN alleviates the hurdle and inflexibility of cable-connected devices for monitoring health conditions via various implantable and wearable sensors.

The IEEE 802.15 TG6 has recently released the WBAN standard that includes the Impulse Radio Ultra Wideband (IRUWB) as its physical layer [9]. Prior to this standard, IEEE also released the WBAN channel models [8] that defined four different channel conditions, i.e. CM1 - CM4, in which CM4 considers the body-to-external link.

Another competing technology for a short range, very high data rate communication is MB-OFDM UWB, endorsed by the WiMedia Alliance [6]. It combines the capability of OFDM to flatten the response of dispersive-frequency selective channels of UWB, while maintaining the benefit of high capacity of UWB. Thus, in [10], we proposed the use of MB-OFDM UWB as an alternative physical layer for WBAN, in combination with Space-Time-Frequency-Code Multi-Input Multi-Output (STFC MIMO) techniques. STFCs are the extended versions of the conventional Space-Time Block Codes (STBCs) to a three -dimensional space, namely space, time and frequency, to increase further the system diversity. Readers may refer to [5] for more details about STFCs. We demonstrated that the proposed system, referred to as STFC MB-OFDM UWB, could provide better performance, higher data rate, and greater range at the price of a modest increase in complexity [10]. Further, we evaluated the performance of the proposed system in the CM4 channel model. One important observation from the result in [10] (or Fig.3 in this paper) is that the system BER performance differs significantly in different body directions, i.e. the direction of the receiver placed on the surface of the body with respect to (w.r.t) the transmitter. This is due to the effects of LOS (Line-of-Sight), partial LOS, and body shadowing. The results are also consistent with the parameters of the IEEE WBAN CM4 channel [8].

Adaptive techniques have been employed for numerous systems and applications in order to enhance their performance. For instance, [2] examined a unified adaptive modulation scheme for a general communication system where the data rate, transmitted power, and instantaneous BER are varied to maximize spectral efficiency. A cross layer adaptive modulation to minimize the transmission energy in wireless sensor networks is proposed in [3]. However, an adaptive scheme for the WBAN physical layer has not been examined. The contribution of this paper is the proposal for the first time a body direction based adaptive algorithm implemented in STFC MB-OFDM UWB WBAN, in order to improve the average BER performance and/or reduce the power consumption of the body-to-external link for WBAN applications. The core idea is that a combination of different digital modulation schemes (BPSK, QPSK), powers of signal constellations, and STFC coding rates is adaptively selected, depending on the body direction w.r.t. the transmitter. The adaption is carried out by the measurement of angles of the body w.r.t. the transmitter. A simple direction sensor, e.g. a Giant Magneto Resistance (GMR) thin film sensor chips, can be used to accurately measure the angle [11]. The angle information is then feedback to the transmitter via a simple feedback loop to vary its modulation, STFC coding rate, and constellation power.

The paper is organized as follows. Section II analyzes the proposed system model, including a review of the CM4 IEEE WBAN channel model. Section III describes the algorithm of the adaptive scheme, including the decoding complexity. Simulation results and analyses are presented in Section IV. Section $\mathrm{V}$ concludes the paper. 


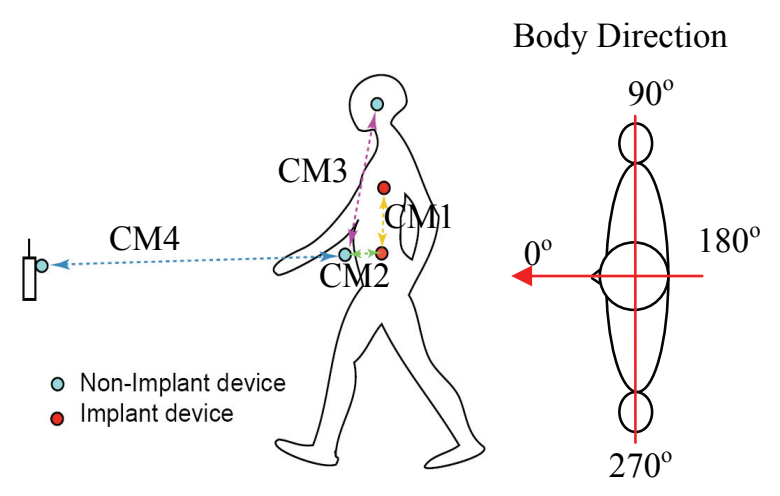

Fig.1. WBAN channel models and body directions of CM4 [8].

\section{SYSTEM MODEL}

\section{A. Review of CM4 IEEE's WBAN Channel Model}

IEEE WBAN CM4 channel model [8] will be used in this paper to create channel realizations for the body surface-toexternal WBAN link as shown in Fig.1. It is applied to three different frequency bands, including the UWB band (3.1-10.6 $\mathrm{GHz}$ ) and takes into account the effect of body directions [8].

The channel response is characterized by the following power decay profile

$$
h(t)=\sum_{m=0}^{L-1} \alpha_{m} \delta\left(t-\tau_{m}\right)
$$

where $\left|\alpha_{m}\right|^{2}=\Omega_{\mathrm{o}} \mathrm{e}^{-\frac{\tau_{\mathrm{m}}}{\Gamma}-k[1-\delta(m)]} \beta$

$$
k=\Delta k\left(\frac{\ln 10}{10}\right) ; \tau_{o}=d / c ; \text { and } \beta \sim \log \operatorname{normal}(0, \sigma) .
$$

$L$ is the number of arrival paths, modeled as a Poisson random variable with the mean value of $400 ; \alpha_{m}$ is the amplitude of each path; $\tau_{m}, m=1, \ldots, L-1$, is timing of path arrivals and is modeled as a Poisson random process with the arrival rate $\lambda=$ $1 / 0.501251 \mathrm{~ns} ; \Gamma$ is an exponential decay factor with $\Omega_{0}$ as the path loss; $k$ is the K-factor (NLOS), $d$ is the $T x$ - $R x$ distance, and $c$ is the velocity of light.

The parameters of CM4 also depend on the direction of body toward the Tx antenna, and listed in the Table I [8].

\section{TABLE I. PARAMETERS OF CM4 [8]}

\begin{tabular}{|c|c|c|c|}
\hline Body Direction & $\Gamma(n s)$ & $k(\Delta k(d B))$ & $\sigma(d B)$ \\
\hline $0^{\circ}$ & 44.6364 & $5.111(22.2)$ & 7.30 \\
\hline $90^{\circ}$ & 54.2868 & $4.348(18.8)$ & 7.08 \\
\hline $180^{\circ}$ & 53.4186 & $3.638(15.8)$ & 7.03 \\
\hline $270^{\circ}$ & 83.9635 & $3.983(17.3)$ & 7.19 \\
\hline
\end{tabular}

From Table I, as opposed to our intuition, the worst link is corresponding to the $270^{\circ}$ body direction, rather than $180^{\circ}$, and the channel behaviours of the $90^{\circ}$ and $270^{\circ}$ are significantly different. It might possibly be due to the asymmetrical radiation pattern of the antenna and different surrounding environments of the $90^{\circ}$, and $270^{\circ}$ directions

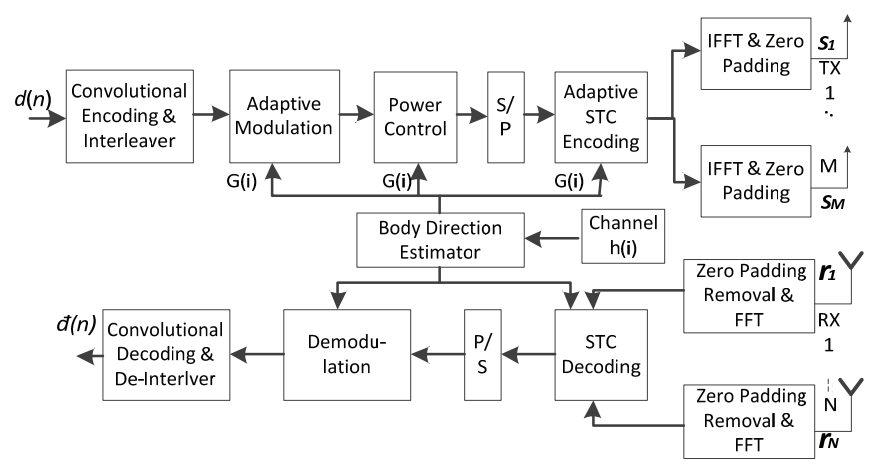

Fig. 2. Adaptive STFC MB-OFDM UWB system

during the measurement. This observation will be reflected in our simulation results in this paper.

\section{B. Proposed Adaptive STFC MB-OFDM UWB System}

Fig.2 depicts the proposed adaptive STFC MIMO-OFDM UWB system with $M$-Tx antennas and $N$-Rx antennas. The data stream $d(n)$ undergoes convolutional coding and interleaving, before being mapped to symbols. The body direction estimator measures the orientation of the body w.r.t. the transmitter. The angular information is then feedback to the transmitter via a simple feedback loop in order to adjust its modulation, constellation power, and STFC coding rate accordingly. The adaptive modulation block selects either QPSK or BPSK, while the power control block adjusts the constellation power based on certain rules as detailed in the next section. Those parameters areadaptively varied in a way that the average total power and total data rate over all four body directions are exactly the same as that in a non-adaptive system for a fair comparison. The stream of modulated symbols is then converted by a Serial-to-Parallel (S/P) block into the symbol blocks (or vectors) $\overline{\mathrm{x}}=\left[x_{1}, x_{2}, \ldots, x_{N_{f f t}}\right]^{T}$, where $N_{f f t}$ is the FFT/IFFT size.

The adaptive STFC block creates a space-time code with either full rate, or 3/2-rate. For the full rate, it uses the Alamouti code [1] to convert the two consecutive symbol blocks, denoted as $\overline{\mathrm{x}}_{1}$ and $\overline{\mathrm{x}}_{2}$, into a STFC block as follows

$$
\mathbf{X}=\left\{\overline{\mathrm{x}}_{t, m}\right\}_{T \times M}=\left[\begin{array}{cc}
\overline{\mathrm{x}}_{1} & \overline{\mathrm{x}}_{2} \\
-\overline{\mathrm{x}}_{2}^{*} & \overline{\mathrm{x}}_{1}^{*}
\end{array}\right]
$$

where $\bar{x}_{1}$ and $\bar{x}_{2}$ are symbol vectors transmitted from the first and the second antenna at a given time slot, respectively. (.) ${ }^{*}$ denotes complex conjugate. For 3/2-rate STFC, three symbol vectors are encoded following the Sezginer-Sari code [4]

$$
\mathbf{X}=\left\{\overline{\mathrm{x}}_{t, m}\right\}_{T \times M}=\left[\begin{array}{cc}
a \overline{\mathrm{x}}_{1}+\frac{b \overline{\mathrm{x}}_{3}}{\sqrt{2}} & -\left(c \overline{\mathrm{x}}_{2}^{*}+\frac{d \overline{\mathrm{x}}_{3}^{*}}{\sqrt{2}}\right) \\
a \overline{\mathrm{x}}_{2}+\frac{b \overline{\mathrm{x}}_{3}}{\sqrt{2}} & c \overline{\mathrm{x}}_{1}^{*}+\frac{d \overline{\mathrm{x}}_{3}^{*}}{\sqrt{2}}
\end{array}\right]
$$


where $a, b, c$, and $d$ are complex-valued design parameters. Here, we use the optimum parameters $a=c=\sqrt{2}$, and $b=d=(1+j \sqrt{7}) / 4$ as determined in [4].

We denote the matrix $\mathbf{X}$ in the general form as $\mathbf{X}=$ $\left\{\overline{\mathrm{x}}_{t, m}\right\}_{T \times M}$, where the index $t$ indicates the time slot and $m$ indicates the Tx antenna. Each of symbol vectors in the matrix $\mathbf{X}$ is then converted into an $N_{f f t}$-point MB-OFDM symbol by the IFFT block, resulting in the STFC code matrix $\mathbf{X}_{O F D M}$ whose elements are the $N_{f f t}$-point IFFT of the corresponding symbol vectors $\overline{\mathrm{x}}_{t, m}$ in $\mathbf{X}$. Hence, the transmitted matrix of STFC MB-OFDM symbols is

$$
\mathbf{X}_{\text {OFDM }}=\left\{\overline{\mathrm{x}}_{O F D M, t, m}\right\}_{T \times M}=\left\{\operatorname{IFFT}\left\{\overline{\mathrm{x}}_{t, m}\right\}\right\}_{T \times M}
$$

The actual transmitted matrix is the matrix $\mathbf{X}_{Z P}$ whose elements are the $N_{f f t}$-length vectors $\overline{\mathbf{x}}_{O F D M, t, m}$ in $\mathbf{X}_{O F D M}$ appended with the 37-samples zero padded suffix (ZPS), denoted as $\bar{x}_{Z P, t, m}$, before being transmitted to the channel. This means

$$
\mathbf{X}_{Z P}=\left\{\overline{\mathbf{x}}_{Z P, t, m}\right\}_{T \times M}
$$

The channels between $M$-Tx antennas and $N$-Rx antennas are defined as the channel matrix $\mathbf{H}$

$$
\mathbf{H}=\left[\begin{array}{ccc}
\overline{\mathrm{h}}_{1,1} & \cdots & \overline{\mathrm{h}}_{M, 1} \\
\vdots & \ddots & \vdots \\
\overline{\mathrm{h}}_{1, N} & \cdots & \overline{\mathrm{h}}_{M, N}
\end{array}\right]
$$

where $\overline{\mathrm{h}}_{m, n}$ is the channel coefficient vector between the $m$-th Tx antenna, for $m=1,2, \ldots, M$, and the $n$-th $\mathrm{Rx}$ antenna, $n=$ $1,2, \ldots, N$, containing $L$ multipath. The distribution and parameters of $\overline{\mathrm{h}}_{m, n}$ are determined by Eq. (1) and Table I.

The received signal at the $n$ - $t h \mathrm{Rx}$ antenna during the $t$-th transmitted OFDM symbol duration is computed as

$$
\overline{\mathrm{r}}_{t, n}=\sum_{m=1}^{M}\left(\overline{\mathrm{x}}_{Z P, t, m} \otimes \overline{\mathrm{h}}_{m, n}\right)+\overline{\mathrm{n}}_{t, n}
$$

where $\otimes$ denotes the linear convolution, $\overline{\mathrm{x}}_{Z P, t, m}$ is the MBOFDM symbol including ZPS (Zero Padded Suffix) transmitted from the $m$-th $\mathrm{Tx}$ antenna, and $\overline{\mathrm{n}}_{t, n}$ is the zero mean additive white Gaussian noise (AWGN) vector. The ZPS is removed by an Overlap-And-Add-Operation (OAAO) prior to the FFT operation. After performing OAAO, the received signal can be written as [5, Eq.(8)]

$$
\overline{\mathrm{r}}_{\text {OFDM }, t, n}=\sum_{m=1}^{M} \overline{\mathrm{x}}_{O F D M, t, m} * \overline{\mathrm{h}}_{m, n}+\overline{\mathrm{n}}_{t, n}
$$

where $(*)$ denotes circular convolution. After the FFT block, the input signals of the STFC decoder is calculated as [5, Eq.(12)]

$$
\overline{\mathfrak{r}}_{t, n}=\sum_{m=1}^{M} \overline{\mathrm{x}}_{t, m} \bullet \overline{\mathfrak{h}}_{m, n}+\overline{\mathfrak{n}}_{t, n}
$$

where $(\bullet$ )denotes the dot (Hardamard) product between the two vectors, $\overline{\mathfrak{r}}_{t, n}=\operatorname{FFT}\left(\overline{\mathrm{r}}_{O F D M, t, n}\right)=\left[\mathfrak{r}_{m, n, 1}, \ldots, \mathfrak{r}_{m, n, N_{f f t}}\right]^{T}$, $\overline{\mathrm{x}}_{t, m}$ is the original modulation symbols, $\overline{\mathfrak{h}}_{m, n}=\operatorname{FFT}\left(\overline{\mathrm{h}}_{m n}\right)=$ $\left[\hbar_{m, n, 1}, \ldots, \hbar_{m, n, N_{f f t}}\right]^{T}, \quad$ and $\quad \overline{\mathrm{n}}_{t, n}=\operatorname{FFT}\left(\overline{\mathrm{n}}_{t, n}\right)$. Denote $\mathcal{R}=\left\{\overline{\mathfrak{x}}_{t, n}\right\}_{T \times N}$ to be the matrix of the received signals after FFT, $\mathcal{H}=\left\{\overline{\mathfrak{h}}_{m, n}\right\}_{M \times N}$ to be the channel response matrix, and $\mathcal{N}=\left\{\overline{\mathfrak{n}}_{t, n}\right\}_{T \times N}$ the matrix of noise. Then we can re-write (9) as

$$
\mathcal{R}=\mathbf{X} \circ \mathcal{H}+\mathcal{N}
$$

where $\left({ }^{\circ}\right)$ denotes the operation which is similar to the normal matrix multiplication, except that each element in $\mathcal{R}$ is determined by (9). Thus the detected vectors are decided by the following Maximum Likelihood (ML) rule

$$
\left\{\tilde{\overline{\mathrm{x}}}_{t, m}\right\}=\arg \min _{\left\{\overline{\mathrm{x}}_{t, m}\right\}}\|\mathcal{R}-\mathbf{X} \circ \mathcal{H}\|_{F}^{2}
$$

Since the matrix $\mathbf{X}$ preserves its orthogonality in the similar manner as in a conventional STBC MIMO system, the STFC MB-OFDM UWB system can also employ a simple linear decoding process. For simplicity, we can omit the time index $t$. Hence in the $2 \mathrm{I} 1 \mathrm{O}$ configuration with M-PSK modulation, we have the following decoding metrics

$$
\begin{aligned}
& \overline{\mathrm{x}}_{1}=\arg \min _{\overline{\mathrm{x}} \in \mathcal{C}^{N_{D}}}\left\|\left(\overline{\mathrm{h}}_{1}^{*} \bullet \overline{\mathfrak{r}}_{1}+\overline{\mathfrak{h}}_{2} \bullet \overline{\mathfrak{r}}_{2}^{*}\right)-\overline{\mathrm{x}}\right\|_{F}^{2} \\
& \overline{\mathrm{x}}_{2}=\arg \min _{\overline{\mathrm{x}} \in \mathcal{C}^{N_{D}}}\left\|\left(\overline{\mathfrak{h}}_{2}^{*} \bullet \overline{\mathfrak{r}}_{1}-\overline{\mathfrak{h}}_{1} \bullet \overline{\mathfrak{r}}_{2}^{*}\right)-\overline{\mathrm{x}}\right\|_{F}^{2}
\end{aligned}
$$

where $N_{D}$ is number of data subcarriers $\left(N_{D}=100\right.$, according to the standard [6]), and $\mathcal{C}^{N_{D}}$ denotes the $N_{D}$-dimensional complex space of the transmitted vector $\overline{\mathrm{x}}$. More importantly, each data point in an MB-OFDM symbol can be decoded separately rather than jointly [5], thus the decoding process is significantly simplified. In particular, the decoding metric of each data at the $k$-th subcarrier $\left(k=1, \ldots, N_{D}\right)$ in the MBOFDM symbols for the $2 \mathrm{I} 1 \mathrm{O}$ configuration are

$$
\begin{aligned}
& \tilde{\mathrm{x}}_{1, k}=\arg \min _{x_{1, k} \in \mathcal{C}}\left[\left|\left(\hbar_{1, k}^{*} \mathfrak{r}_{1, k}+\hbar_{2, k} \mathfrak{r}_{2, k}^{*}\right)-x_{1, k}\right|^{2}\right] \\
& \tilde{\mathrm{x}}_{2, k}=\arg \min _{x_{2, k} \in \mathcal{C}}\left[\left|\left(\hbar_{2, k}^{*} \mathfrak{r}_{1, k}-\hbar_{1, k} \mathfrak{r}_{2, k}^{*}\right)-x_{1, k}\right|^{2}\right]
\end{aligned}
$$

Similarly, for the $2 \mathrm{I} 2 \mathrm{O}$ configuration, the decoding metrics are

$$
\begin{aligned}
& \tilde{\mathrm{x}}_{1, k}=\arg \min _{x_{1, k} \in \mathcal{C}}\left[\left|\sum_{n=1}^{2}\left(\hbar_{1, n, k}^{*} \mathfrak{r}_{1, n, k}+\hbar_{2, n, k} \mathfrak{r}_{2, n, k}^{*}\right)-x_{1, k}\right|^{2}\right] \\
& \tilde{\mathrm{x}}_{2, k}=\arg \min _{x_{2, k} \in \mathcal{C}}\left[\left|\sum_{n=1}^{2}\left(\hbar_{2, n, k}^{*} \mathfrak{r}_{1, n, k}-\hbar_{1, n, k} \mathfrak{r}_{2, n, k}^{*}\right)-x_{2, k}\right|^{2}\right]
\end{aligned}
$$

For the 3/2-rate STFC, we follow the decoding process as mentioned in [4]. Generalization for the case of $M$-Tx and 


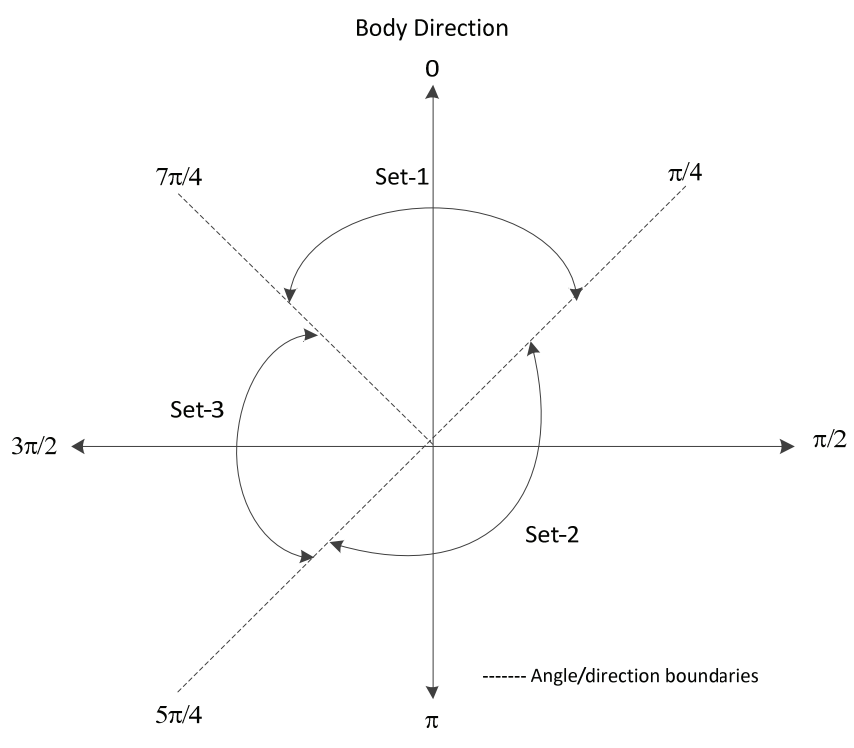

Fig.3. Angle direction boundaries for adaptive decision.

$N$-Rx antennas is straightforward.

\section{ADAPTIVE SCHEME}

\section{A. Adaptive Selection Algorithm}

As mentioned previously in Section I, adaptive modulation and coding schemes have not been examined for WBANs using MB-OFDM UWB technology, though they have been intensively researched for other systems. This section proposes for the first time a simple-but-efficient adaptive modulation and space-time-frequency coding scheme for such system. The proposed adaptive scheme is controlled by the measurement of the angular direction of the body. Depending on which region among three regions shown in Fig. 3 the current body direction belongs to, the corresponding 2-bit angular information is fed back to the transmitter to select a suitable combination of its modulation, STFC coding rate, and constellation power. It is noted that the three regions in Fig. 3 have been derived based on our observations that the best channel behaviour is corresponding to the $0^{\circ}$ direction, the $90^{\circ}$ and $180^{\circ}$ directions possess relatively close error performances, and the worst channel behaviour is the $270^{\circ}$ direction. These observations have been discussed in our previous publication [10], and can also be seen in Fig. 4 mentioned in Section IV of this paper.

Either QPSK or BPSK is used for modulation. We use two different STFC coding rates, namely coding rate-1 which uses an Alamouti full rate code, and coding rate-3/2 which uses a Sezginer-Sari $3 / 2$ rate code. The normalized transmitted constellation power may take one of the three values $0.5,1.0$, and 1.5. The selection of the adaptive scheme is done in a way that the average data rate and total transmitted power over all main four directions of the body are maintained exactly equal to those in a non-adaptive system (rate-1.0 STFC, constellation power 1.0, and QPSK modulation for all four directions) for a fair comparison. This is done as follows.
1. Data rate constraint: the lowest density signal constellation (BPSK) is used for the worst link $\left(270^{\circ}\right)$ in order to improve the system error performance over this direction. A higher density constellation (QPSK) is used for all other three directions. The highest rate STFC (rate-3/2) is selected for the best link $\left(0^{\circ}\right)$ while the rate- 1 STFC is selected for all three remaining directions. Thus the average spectrum efficiency over four main directions is maintained at $2 \mathrm{bits} / \mathrm{s} / \mathrm{Hz}$ which is exactly the same as that in the non-adaptive system.

2. Power constraint: the normalized power of one is selected for the signal constellations (QPSK) for the $90^{\circ}$ and $180^{\circ}$ directions, similar to the non-adaptive system, while the highest power level (1.5) is selected for the best link $\left(0^{\circ}\right)$ to compensate for the possible performance degradation caused by the highest STFC coding rate $(3 / 2)$ chosen for this direction. The above power allocation leaves the power of 0.5 for the BPSK signal constellation in the $270^{\circ}$ direction. Thus the total transmitted power over all four main directions is exactly the same as that in the non-adaptive system.

The core idea behind the proposed algorithm is that the best channel conveys the most information with the most power allocated while the worse link carries the least information. Hence, we define three set of adaptive schemes. Set- 1 is aimed to take advantage of best channel link by maximizing the capacity, i.e. by using QPSK modulation and STFC code rate $3 / 2$ and normalized power TX as 1.5 . Set-2 uses QPSK, STFC code rate 1.0, and power TX 1.0, and Set-3 uses BPSK, STFC code rate 1.0, and power TX 0.5.The proposed algorithm is summarized as follows:

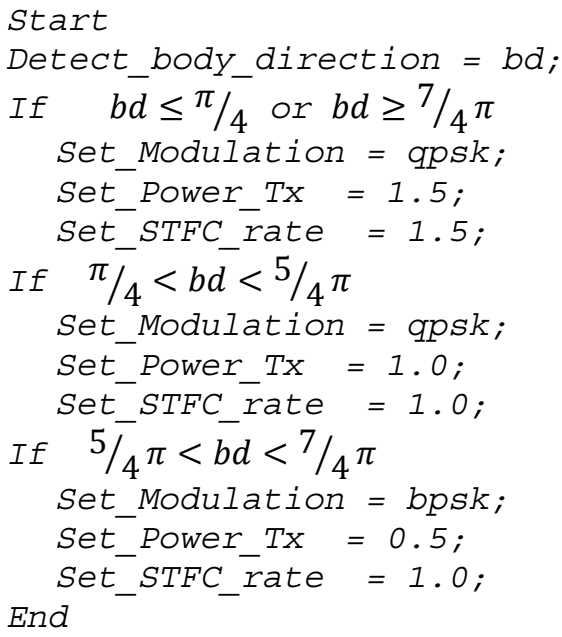

\section{B. Decoding Complexity}

The decoding process of the $3 / 2$ rate code has the complexity $O(4)$ compared with the full rate code, since the decoder firstly has to decide $\tilde{\mathrm{x}}_{3}$ from four possible symbols in QPSK, prior to decoding $\tilde{\mathrm{x}}_{1}$ and $\tilde{\mathrm{x}}_{2}$ symbols. Meanwhile, in the worst link, the complexity is reduced to $O(2)$ due to BPSK (rather than QPSK) is used. The overall complexity of the decoding process increases by a factor of $[O(4)-O(2)]$, beside the body direction estimation. It should be emphasized that, though the decoding complexity in the adaptive system increases, the decoding processes for both adaptive and nonadaptive system are relatively simple. 
For body direction estimation, a simple direction sensor, e.g. by using a Giant magneto resistance (GMR) thin film sensor chips, can be used. It is a magnetic sensor, robust, and capable to provide $360^{\circ}$ angular measurement [11]. As mentioned above, the measured body direction is not fed back directly to the transmitter. Instead, depending on which region among the three pre-defined regions shown in Fig. 3 this body direction belongs to, two bits are required to be fed back to the transmitter to indicate this region in order for the transmitter to select the corresponding combination of modulation, STFC structure, and constellation power. In other words, the proposed adaptive scheme could be implemented with a slightly increased system complexity.

\section{Simulation Results}

The Monte-Carlo simulations are carried out to compare the average BER performance of the adaptive system with that of the non-adaptive one, assuming perfect channel state estimations are available at the receiver. The non-adaptive system, as the benchmark, uses QPSK modulation, normalized power of 1.0, and the full rate STFC for all body directions. It is assumed that a person wearing the WBAN devices moves and turns the body clock-wisely against the transmitter. Channel coefficients are assumed to be constant during each

TABLE II. SIMULATION PARAMETERS.

\begin{tabular}{|c|c|}
\hline Parameters & Value \\
\hline FFT \& IFFT size $N_{f f t}$ & 128 \\
\hline Number of ZPS $N_{\text {ZPS }}$ & 37 \\
\hline Convolutional coder $(\mathrm{K}=7)$ rate & \multicolumn{1}{|c|}{ Viterbi, Hard } \\
\hline Conv. decoder and mode & $\begin{array}{l}\text { Column-wise written, row- } \\
\text { wise read }\end{array}$ \\
\hline Interleaver/De-interleaver & 400 \\
\hline Average number of paths in CM4 & \\
\hline
\end{tabular}

STFC block, but random between consecutive STFC blocks. The channel realizations are simulated by the Matlab ${ }^{T M}$ program enclosed in the appendix of IEEE 802.15.6 channel modeling subcommittee final document [8]. Other simulation parameters are listed in Table II.

Fig. 4 shows the performance of a non-adaptive system for both $2 \mathrm{I} 1 \mathrm{O}$ and $2 \mathrm{I} 2 \mathrm{O}$ configurations. It reveals a significant degradation in the BER performance within almost the whole range of SNR when the receiver (on the body) turns away from the transmitter. The front body has a LOS component, resulting in the best performance compared to other directions of the body. The back of the body $\left(180^{\circ}\right.$ direction) suffers from a body shadowing effect and only receives NLOS multipath signals. The performance is still reasonably good in the very dispersive channel, particularly with the $2 \mathrm{I} 2 \mathrm{O}$ configuration. Its performance degrades $6 \mathrm{~dB}$ for $\mathrm{BER}=10^{-4}$, compared to the front body.

Fig. 4 also shows that, the $270^{\circ}$ direction experiences a significant performance degradation, compared to the $90^{\circ}$ direction. This is possibly due to the difference of side lobes of the Rx antenna when it turns $90^{\circ}$ and $270^{\circ}$ and/or the

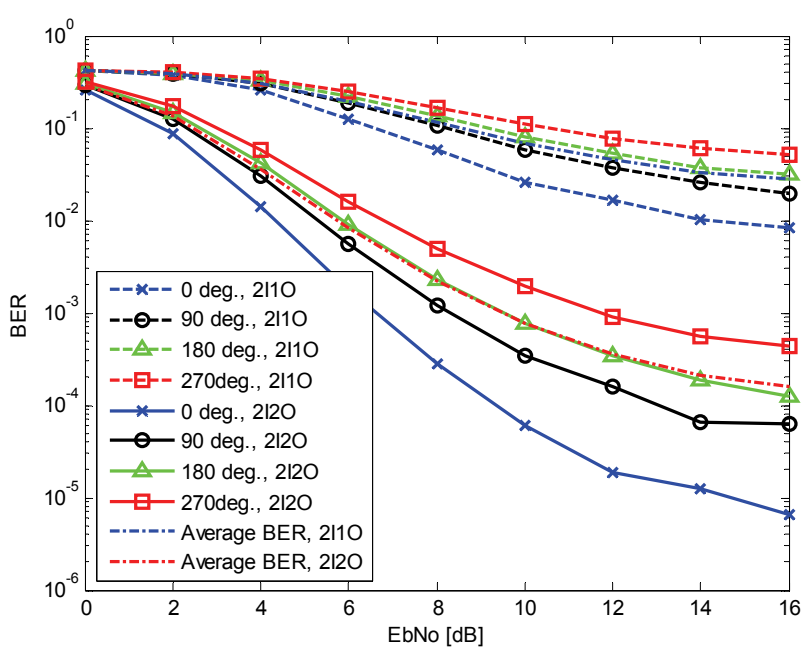

Fig.4. Performance of non-adaptive scheme in CM4.

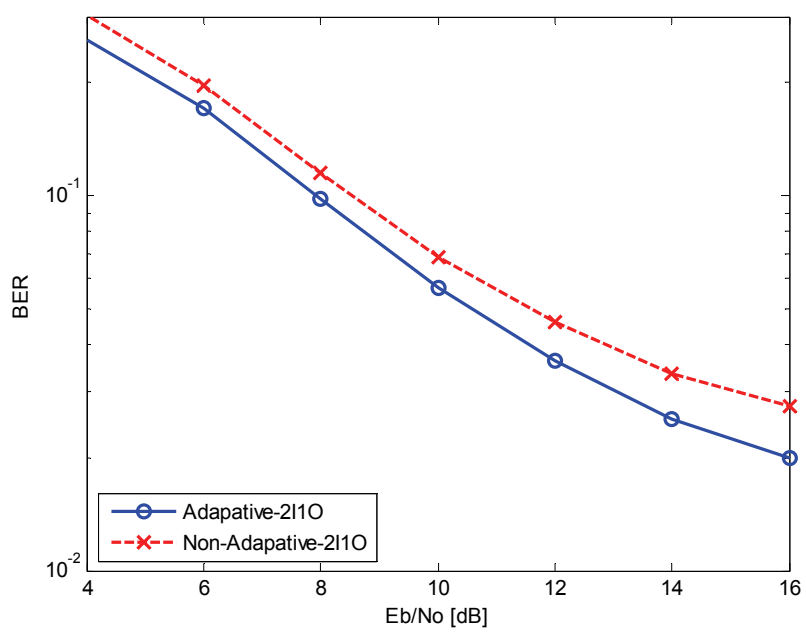

(a)

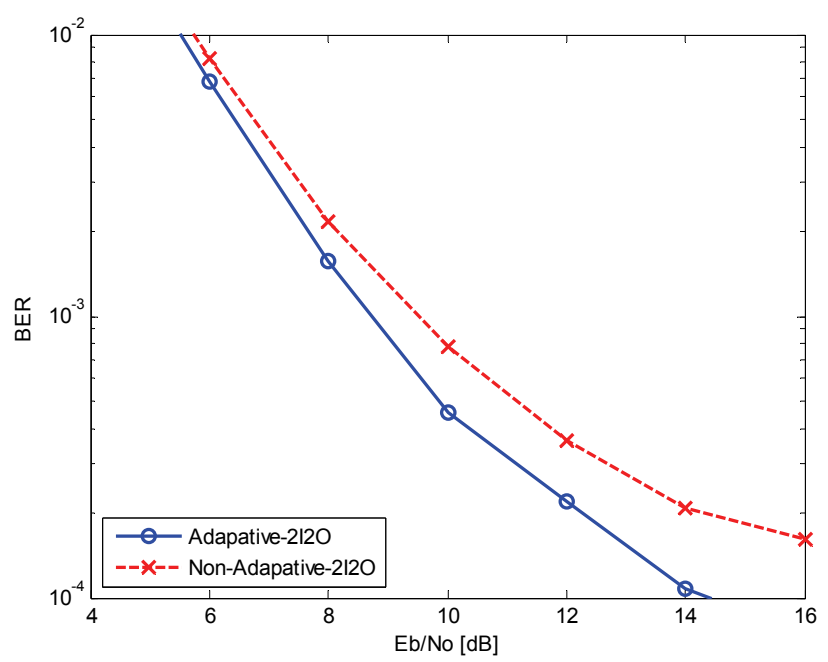

(b)

Fig.5. Performances of adaptive scheme in CM4 (a) $2 \mathrm{I} 1 \mathrm{O}$, (b) $2 \mathrm{I} 2 \mathrm{O}$. 
propagation environment surrounding these directions. This observation is consistent with parameters of CM4 as mentioned in Table I. The average BER performance for each MIMO configuration is then calculated over four body directions and used as the benchmark for comparison with the adaptive system. This average BER performance is shown by dotted curves in Fig.4 for the ease of comparison with the proposed adaptive algorithm.

Fig. 5 shows the improvement of the adaptive scheme compared to the non-adaptive one in $2 \mathrm{I} 1 \mathrm{O}$ and $2 \mathrm{I} 2 \mathrm{O}$ configurations. The BER averaged over all four directions in the adaptive scheme shown by a circled-continuous line is consistently better than that of the non-adaptive one in a $2 \mathrm{I} 1 \mathrm{O}$ configuration by about $1-2 \mathrm{~dB}$ in the whole SNR region, and by about $1-3 \mathrm{~dB}$ in the medium-to-high SNR region in a $2 \mathrm{I} 2 \mathrm{O}$ configuration. If we compare the average BER of the adaptive scheme with the BER of the worst scenario $\left(270^{\circ}\right.$ direction) in the non-adaptive one (cf. Square-marked curves in Fig.4), the improvement is significantly high, i.e. $5.5 \mathrm{~dB}$ at BER $5 \cdot 10^{-2}$ in the $2 \mathrm{I} 1 \mathrm{O}$ configuration and $6 \mathrm{~dB}$ at BER $4 \cdot 10^{-4}$ in the $2 \mathrm{I} 2 \mathrm{O}$ case. (Comparing to the BER of the best link $\left(0^{\circ}\right.$ direction, cross-marked curves in Fig.4) of the non-adaptive system in both MIMO configurations, as expected, the averaged BER of the adaptive scheme is slightly worse due to the fact that, the adaptive system selects the highest STFC coding rate $(3 / 2)$ for the $0^{\circ}$ direction in order to maximize the capacity, with a price of minor performance degradation). It is noted that the aforementioned average BER improvements are achieved without any increase of the total transmitted power or any sacrifice of the data rate. Due to the fact that power is the main constraint in WBAN applications [7], [9], an improvement in the order of $1-3 \mathrm{~dB}$ means a reduction of $12.5 \%-50 \%$ of the total transmitted power, while maintaining the same BER performance as in the non-adaptive system.

\section{CONCLUSION}

We have presented a novel body direction based adaptive STFC MB-OFDM UWB WBAN system, in order to improve the average BER performance and/or reduce the power consumption. Simulation results confirm that the proposed system can achieve a consistent $1-2 \mathrm{~dB}$ improvement in the case of 2110 , and approximate $1-3 \mathrm{~dB}$ in the medium-to-high SNR range in the $2 \mathrm{I} 2 \mathrm{O}$ configuration compared to the nonadaptive system. Those improvements practically mean a possible reduction of $12.5 \%$ - $50 \%$ of the total transmitted power. In other words, it can save and prolong significant battery life of WBAN devices. We conclude that, with the price of slightly increased complexity, the proposed system can provide a power saving and better average BER performance for WBAN applications without sacrificing the data rate. Our future work will focus on the adaptive scheme driven by the measured BER in the receiver.

\section{ACKNOWLEDGMENT}

The first author is grateful for the funding support from AusAID through Australian Development Scholarship.

\section{REFERENCES}

[1] S. M. Alamouti, "A simple transmit diversity technique for wireless communications," IEEE Journal on Selected Areas in Communications, vol. 16, no. 8, pp. 1451-1458, Oct. 1998.

[2] S. T. Chung and A.J. Goldsmith, "Degrees of freedom in adaptive modulation: a unified view", IEEE Trans. On Commun., Vol. 49, No. 9,pp. 1561- 1571, Sep. 2001.

[3] J. Garzas, C. B. Calzon, and A. G. Armada, "An energy-efficient adaptive modulation suitable for wireless sensor networks with SER and throughput Constraints", EURASIP Journal on Wireless Commun. and Netw., vol. 2007, pp. 1 - 7, April 2007.

[4] S. Sezginer and H. Sari, "A High-Rate Full-Diversity $2 \times 2$ Space-Time Code with simple Maximum Likelihood decoding", Proc. ISSPIT'07, pp. 1132-1136, Dec. 2007.

[5] L.C. Tran and A. Mertins, "Space-Time-Frequency Code implementation in MB-OFDM UWB communications: design criteria and performance", IEEE Trans. Wireless Communication, vol. 8, no. 2, Feb. 2009.

[6] A. Batra et al., "Multiband OFDM physical layer specification," WiMedia Alliance, Final Deliverable 1.5, Aug. 2009.

[7] M. Chen, et al., "Body Area Networks: A Survey", Springer Journal on Mobile Netw Appl, vol. 16, no. 2, pp.171-193, Aug. 2010.

[8] K.Y. Yazdandoost and K. Sayrafian-Pour , " Channel model for Body area network (BAN) “, IEEE P802.15-08-0780-12-0006, Nov. 2010.

[9] IEEE Computer Society, “ IEEE standard for local and metropolitan area networks-Part 15.6: Wireless Body Area Networks”, Feb. 2012.

[10] M. Sudjai, L.C.Tran, and F. Safaei, "Performance analysis of STFC MBOFDM UWB in WBAN channels", Proc. 23rd IEEE PIMRC'12, pp. 1704-1709, Sept., 2012.

[11] C.P.O. Treutler, "Magnetic sensors for automotive applications", Elsevier Journal on Sensors and Actuator, vol. A91 , pp. 2-6, 2001. 